\title{
EL ESTUDIO DE CASO EN LA INVESTIGACIÓN CUALITATIVA
}

\author{
MARÍA MARTHA DURÁN \\ Universidad Estatal a Distancia, Costa Rica \\ mduranr@uned.ac.cr
}

\section{RESUMEN}

Dada la importancia y uso del Estudio de Caso en la investigación cualitativa desde los años 70 por su ventaja epistemológica sobre otros métodos de indagación, se discutirá en este artículo acerca de las características y requerimientos de su unidad de análisis. Se propone además un concepto de Estudio de Caso Cualitativo y se delimita el uso en cuatro diferentes situaciones. Por último se presentan las fortalezas y limitaciones respecto a su uso en investigación cualitativa y el impacto en el quehacer investigativo.

PALABRAS CLAVE: INVESTIGACIÓN CUALITATIVA, ESTUDIO DE CASO, REQUERIMIENTOS, LIMITACIONES

\section{ABSTRACT}

Given the importance and use of Case Studies in qualitative research since the 70's, this article discusses the characteristics and requirements of this technique. It also proposes a concept of ECC and outlines the use of four different situations. Finally, the article reviews the strengths and limitations regarding its use in qualitative research and its impact on the research process in general.

KEYWORDS: QUALITATIVE RESEARCH, CASE STUDIES, REQUIREMENTS, LIMITATIONS

\section{INTRODUCCIÓN}

El Estudio de Caso (EC) es una forma de abordar un hecho, fenómeno, acontecimiento o situación particular de manera profunda y en su contexto, lo que permite una mayor comprensión de su complejidad y, por lo tanto, el mayor aprendizaje del caso en estudio. Utiliza múltiples fuentes de datos y métodos, es transparadigmático y transdisciplinario.

En la Investigación Cualitativa, el Estudio de Caso tiene una ventaja epistemológica sobre otros métodos de indagación (Stake, 1978, 2005) pues se considera útil en el estudio de asuntos humanos dada su flexibilidad y porque logra captar la atención de diferentes lectores gracias a un estilo accesible, frecuentemente en armonía con la experiencia del lector, constituyéndose en una base natural de generalización. Además por la universalidad e importancia del conocimiento experiencial y la compatibilidad del EC con este.

Sin embargo esta utilización en investigación ha generado importantes problemas focalizados especialmente en la utilización sin fundamento ni conocimiento, y la pérdida de claridad sobre su concepto. Esto a su vez han incidido en las fuertes críticas en relación a su validez, confiabilidad y posibilidad de generalización de conocimiento, aspectos enlazados a los mitos que Flyvbjerg propone (Flyvbjerg, 2003; VanWynsberghe y Kahn, 
2007): 1. el conocimiento teórico es más valioso que el conocimiento práctico; 2. no es posible generalizar a partir de un solo caso y, por lo tanto, el estudio de un solo caso no puede contribuir al desarrollo científico; 3. los estudios de caso son más útiles para generar hipótesis, mientras otros métodos son más adecuados para verificar las hipótesis y construir las teorías; 4. el estudio de caso contiene un sesgo hacia la verificación, y 5. suele ser difícil resumir estudios de caso específicos.

En este artículo se presentará la Investigación Cualitativa y la relación del Estudio de Caso, características y requerimientos de su unidad de análisis. Se evidencian los problemas de delimitación del concepto y el impacto en el quehacer investigativo.

\section{La investigación cualitativa}

Al descomponer el concepto "Investigación Cualitativa" nos acercamos a una forma de buscar conocimiento, manipularlo y aplicarlo en la realidad concreta, en diferentes niveles y profundidades. La palabra "cualitativa" implica un énfasis en las cualidades o características de entidades, en sus procesos y significados.

La investigación cualitativa es un campo interdisciplinar, transdiciplinar y en muchas ocasiones contradisciplinar. Atraviesa las humanidades, las ciencias sociales y las físicas. La investigación cualitativa es muchas cosas al mismo tiempo. Es multiparadigmática en su enfoque. Los que la practican son sensibles al valor del enfoque multimetódico. Están sometidos a la perspectiva naturalista y a la comprensión interpretativa de la experiencia humana. Al mismo tiempo, el campo es inherentemente político y construido por múltiples posiciones éticas y políticas (Nelson et al., 1992, p4., citado por Denzin y Lincoln, 2005, p. 7).
Para Denzin y Lincoln (2005) y Gurdián (2010) la investigación cualitativa es una actividad que localiza al observador en el mundo; consta de una serie de prácticas interpretativas, materiales que hacen al mundo visible y lo transforman, convirtiéndolo en una serie de representaciones, incluyendo anotaciones de campo, entrevistas, conversaciones, fotos, grabaciones, entre otros. En este nivel, la investigación cualitativa involucra un planteamiento naturalista interpretativo del mundo, lo que significa que los investigadores cualitativos estudian cosas en su ambiente natural, tratando de darle sentido, o interpretar los fenómenos en términos del significado que las personas (los actores) les dan. Para esto los investigadores cualitativos utilizan una gran variedad de prácticas interpretativas interconectadas en procura de obtener una mejor comprensión del tema en estudio en contextos variados y complejos, asumiendo que cada práctica hace al mundo visible de una forma diferente pues la realidad objetiva nunca puede ser captada, de manera que conocemos una cosa sólo a través de sus representaciones.

Por esto la investigación cualitativa esta inherentemente enfocada en lo multimetódico (Flick, 2002, pp. 226-227, citado por Denzin y Lincoln, 2005, p. 5). El uso de múltiples métodos (triangulación) refleja un intento para obtener una profunda comprensión del fenómeno en cuestión convirtiéndose en una alternativa para la validación al añadir rigor, extensión, complejidad, riqueza, y profundidad a cualquier investigación. Por esto, Gurdián (2010) señala que "los procesos de investigación cualitativa son de naturaleza multicíclica y en espiral. Responden generalmente a un diseño semiestructurado y flexible. Esto implica que las hipótesis de trabajo o supuestos tienen un carácter emergente y que evolucionan dentro de una dinámica heurística" (p.180). 
Para Gurdián (2010) la principal característica de la investigación cualitativa es su interés por captar la realidad a través de los ojos de los sujetos actuantes, a partir de la percepción que ellos y ellas tienen de su propio contexto, asumiendo que la realidad se construye socialmente, es histórica y cambia constantemente. Así se intenta la construcción de un tipo de conocimiento, que permita captar el punto de vista de quienes producen y viven la realidad social y cultural, entendiendo que el acceso al conocimiento (de lo específicamente humano), se relaciona con un tipo de realidad epistémica cuya existencia transcurre en los planos de lo subjetivo y lo inter-subjetivo y no solo de lo objetivo. Para la autora esto se traduce en la adopción de una postura metodológica de carácter dialógico en la que las creencias, los valores, los mitos, los prejuicios y los sentimientos, entre otros, son aceptados como elementos de análisis para producir conocimiento sobre la realidad humana (2010).

Gurdián (2010, pp. 66-68) y Ceballos (2009, p 416) se refieren a una serie de supuestos o niveles interdependientes que acompañan el proceso investigativo en general y que se detallan para la investigación cualitativa:

1. Fundamentos o supuestos ontológicos: se refieren a la visión de mundo que tiene la investigadora o el investigador, el concepto de realidad-realidades, a su dinámica y complejidad, en la que subyace el proceso investigativo y del que dependerá el tipo de problemas que se plantean, la perspectiva desde la cual se les aborda y la forma en que se trata de buscar respuestas.

2. Referencia o supuestos epistemológicos: refiere al modelo de relación que seleccione la investigadora-el investigador para relacionarse con lo investigado. Es decir, la for- ma en que sobre la base de determinados principios se adquiere el conocimiento. Desde la investigación cualitativa se busca minimizar la distancia o separación objetiva entre el investigador y aquéllos a quienes estudia para lo cual quien investiga interactúa con las personas observándolas por un período prolongado, viviendo o colaborando con ellas.

3. Fundamentos o supuestos metodológicos: Se refieren a la forma en que enfocamos los problemas, interrogantes o situaciones y les buscamos las respuestas. También comprende el procedimiento, la identificación y selección de las fuentes de donde vamos a obtener la información que buscamos, las técnicas e instrumentos de recolección y de análisis de los datos. Es el modo en que podemos obtener los conocimientos de la realidad que investigamos.

Ceballos (2009) agrega dos niveles de interés desde nuestra perspectiva:

- El supuesto axiológico, que refiere a que en la investigación cualitativa se admite que aunque se clarifiquen las descripciones y se dé solidez a las interpretaciones, la recolección y la interpretación de los datos están influenciadas por la experiencia e intención del investigador, por lo cual es necesario reportar de manera activa esos juicios y valores propios, esto es, hacer a los demás conscientes de ellos.

- El elemento retórico, que en este contexto remite a la narrativa personal y literaria (metáforas, uso del pronombre personal "yo", etc.) y cuenta con un glosario de términos emergentes que son importantes marcadores al momento de escribir (comprensión, significado, etc.). El lenguaje se basa en definiciones que emergen de los 
informantes, en vez de ser definido por el investigador. Este aspecto está relacionado con lo que Stake (2005, pp. 455-456) identifica como storytelling en referencia a la transferencia de conocimiento del investigador al lector y las diferentes formas de realizarlo.

En este entorno, tal como Denzin y Lincoln (2005, p.7) lo señalan, el investigador cualitativo se somete a una doble tensión simultáneamente: por una parte, es atraído por una amplia sensibilidad, interpretativa, postmoderna, feminista y critica; por otra, puede serlo por unas concepciones más positivistas, postpositivistas, humanistas y naturalistas de la experiencia humana y su análisis.

Denzin y Lincoln (2005) proponen que la imagen central para la investigación cualitativa debería ser el cristal, no el triángulo (referido a la triangulación de datos) pues los cristales crecen, cambian, se alteran... son prismas que reflejan externalidades y las refractan dentro de ellos, creando colores diferentes, patrones, arreglos, desplegándolos en diferentes direcciones. Sin embargo creo que la metáfora del holograma' parece mas apropiada: refleja la complejidad de un caso, a partir del paso de un pensamiento lineal, a uno interactivo y complejo, integrado e interrelacionado que permite evidenciar las relaciones que unen cada

1 El holograma es, en términos muy generales, "una fotografía tridimensional producida por medio de un rayo láser en dos partes. Una de las partes del rayo es dirigido al objeto y reflejado en un plato fotográfico de alta resolución. El otro rayo (el de referencia) es proyectado directamente en el plato El patrón que forma la intersección de los dos rayos se graba en el plato. Cuando se ilumina por detrás (en la misma dirección que el rayo de referencia) se logra la proyección en el espacio de una imagen tridimensional del objeto, el cual va cambiando de perspectiva si se mira de distintos ángulos." (Badilla, 2009, p. 11) elemento o dato del caso, considerando perspectivas diferentes según el contexto de los actores en la investigación.

Con estos elementos de base se presentará el Estudio de Caso en la Investigación Cualitativa.

\section{El Estudio de Caso}

En Escuelas de Negocios o en clínicas médicas, en Derecho, Psicología, Antropología o Educación, el Estudio de Caso se utiliza de manera intensiva especialmente desde los años 70, de dos formas: como herramienta pedagógica o como forma de investigar. Escudero et al (2008) los identifica como:

- Método de enseñanza-aprendizaje

"un estudio de caso es un método de aprendizaje, que parte de un ejemplo complejo considerado como un todo en su contexto, y se basa en la comprensión en conjunto (holístico) de dicho ejemplo, a partir de una descripción y un análisis muy detallados" (United States General Accounting Office, 1990, p 14, citado por Escudero et.al, 2008, p. 8).

El estudio de caso en Educación, por ejemplo, imita o simula una situación real (Ellet, 2007, p.13), asumiendo que el Caso es una representación verbal de la realidad que pone al lector en el rol de un participante en la situación. Aunque la unidad de análisis del caso varía enormemente (de un individuo singular u organización, a una nación entera o el mundo), tiene un propósito común: representar la realidad, transmitiendo una situación con todas sus complicaciones y asperezas (incluyendo irrelevancias, facetas, ideas equivocadas, poca o mucha información sobre ella). Se presenta de manera lógica y coherente, fluida pero inevi- 
tablemente involucra incertidumbre pues las situaciones reales consisten en alguna claridad, mucha o muy poca información, y muchas contingencias. Y eso hacen los casos: proveen a los estudiantes del equivalente de un laboratorio usado por los médicos en su formación.

\section{- Estrategia de investigación}

Es "una investigación empírica que estudia un fenómeno contemporáneo dentro de su contexto de la vida real, especialmente cuando los límites entre el fenómeno y su contexto no son claramente evidentes" (Yin, 1994, pág. 13, citado por Escudero et al, 2008, p.8).

Una investigación de estudio de caso trata exitosamente con una situación técnicamente distintiva en la cual hay muchas más variables de interés que datos observacionales; y, como resultado, se basa en múltiples fuentes de evidencia, con datos que deben converger en un estilo de triangulación; y, también como resultado, se beneficia del desarrollo previo de proposiciones teóricas que guían la recolección y el análisis de datos.

\section{El Estudio de Caso en la Investigación Cualitativa}

Para la investigación cualitativa, el Estudio de Caso ha tenido gran importancia en el desarroIlo de las Ciencias Sociales, de la Salud y la Educación. Los estudios de Freud, Piaget, Maslow y Rogers - entre otros- que nacieron y se nutrieron del Estudio de Caso, son algunos ejemplos de ese aporte y relevancia. Dos estudios de caso que son referentes importantes son los estudios de "El niño salvaje de L'Aveyron" (Itard, 1801-1806) y el "Caso de Sydney Blotzman" (Shaw, 1931) ${ }^{2}$.

En las décadas de 1970 y 1980 el Estudio de Caso tuvo un surgimiento muy importante entre los investigadores de la época; ciencias como la Pedagogía y la Psicología lo favorecieron como base metodológica para desarrollar investigaciones principalmente de corte cualitativo.

Stake (2005) señala que el Estudio de Caso es una forma común de hacer investigación cualitativa. Ya en 1978 este autor, uno de los pioneros en la aplicación de Estudio de Caso, señalaba que su popularidad se debía a su estilo y a su utilidad para la exploración de quienes buscan leyes explicativas. Además por la universalidad e importancia del Conocimiento Experiencial y la compatibilidad de los Estudios de Caso con este.

Dado sus múltiples usos, el significado y rigurosidad metodológica del Estudio de Caso ha sufrido daños importantes. Por ejemplo el señalamiento indiscriminado de Estudio de Caso como enfoque, estrategia, método o metodología, diseño de investigación (VanWynsberghe y Khan, 2007) y su impacto en la confusión y pérdida de significados, como se refleja en la tabla 1.

2. La historia natural de una carrera de delincuente (The Natural History of a Delinquent Career; Clifford Shawn, en colaboración con Maurice Moore, 1931) tuvo como objetivo el estudiar a fondo un único caso (el de Sydney Blotzman y las causas que llevaron a este adolescente a cometer actos delictivos) para que sirviera de ilustración prototípica de un determinado problema que, de otro modo, no podría ser estudiado tan en profundidad. 


\section{TABLA 1}

\section{EJEMPLOS DE DEFINICIONES DE EC SEGÚN SU ABORDAJE}

\begin{tabular}{|c|c|}
\hline Uso & Ejemplo \\
\hline $\begin{array}{l}\text { EC como } \\
\text { método }\end{array}$ & $\begin{array}{l}\text { "El Estudio de Casos, un método actual en investigación... } \\
\text { "Hoy en día es uno de los métodos investigativos utilizado con mayor frecuencia por aquellos investigadores acuciosos, dedicados a profundizar en la } \\
\text { naturaleza de un fenómeno dado. En este sentido Grunow (1995) destaca la importancia de esta metodología al revisar } 204 \text { estudios empíricos y encon- } \\
\text { trar que en el 50\%de estos estudios se adoptó esta metodología de investigación" (González, 2009, p. 307). }\end{array}$ \\
\hline $\begin{array}{l}\text { EC como } \\
\text { metodología }\end{array}$ & $\begin{array}{l}\text { "Qualitative case study methodology provides tools for researchers to study complex phenomena within their contexts (Baxter y Jack, 2008, p. 544). } \\
\text { "The case study methodology is also consistent with the constructionist epistemology and constructivist theoretical perspective in that it"will put all understan- } \\
\text { dings, scientific and nonscientific alike, on the very same footing"(Crotty, 1998, p. 16, citado por Marrero et al, 2010, p.82). } \\
\text { "This analysis will be explored within the context of a case study research methodology as defined by Yin (2003) and Stake (1995, 2000). This research } \\
\text { methodology is appropriate when one is considering" how" and "why" questions, particularly over a period of time, as is the case with this example" (Yin, } \\
\text { 2003, citado por Siemens, 2010, p.77) } \\
\text { "We have used the case study methodology proposed by Yin (1984). By using this methodology the researcher can conduct its"participant observation" } \\
\text { research in its working context" (Reis et al, 2010, pp 111-112). }\end{array}$ \\
\hline $\begin{array}{l}\text { EC como } \\
\text { enfoque }\end{array}$ & $\begin{array}{l}\text { "Algunos autores utilizan el término "estudio de casos" para referirse a un método de investigación. . . mientras que otros lo emplean para denominar al } \\
\text { enfoque con el que se realizan ciertas investigaciones... } \\
\text { La investigación con enfoque de estudio de casos pertenece al paradigma cualitativo interpretativo" (Ceballos, 2009, p.416). } \\
\text { "The case study approach was utilized because the researchers believe it is better able to help them understand the culturally responsive teaching of } \\
\text { science teachers as they relate to culture, race, and ethnicity of -0thers' (Stake, 1994)" (Atwater et al, 2010, p.293). }\end{array}$ \\
\hline $\begin{array}{l}\text { EC como } \\
\text { estrategia }\end{array}$ & $\begin{array}{l}\text { "Una estrategia de indagación comprende muchas habilidades, suposiciones, y prácticas que el investigador emplea a medida que se mueve desde el } \\
\text { paradigma hacia el mundo empírico . . ponen en movimiento los paradigmas de interpretación. . . (y) conectan al investigador con métodos específicos } \\
\text { para colectar y analizar materiales empíricos. Por ejemplo, la estrategia del estudio de casos depende de entrevistar, observar, y analizar documentos...." } \\
\text { (Denzin y Lincoln, 2005, p.25) } \\
\text { "This study employs an embedded case study (Yin, 1994) as a strategy for studying a unique department that is heavily involved in academic capitalism } \\
\text { and in which the faculty members themselves are the main source of empirical evidence" (Mendoza y Berger, 2008, p.4). }\end{array}$ \\
\hline Mezclados & $\begin{array}{l}\text { "La estrategia de investigación de estudio de caso es el método más adecuado cuando se plantea en el estudio una pregunta de investigación donde su } \\
\text { forma es del tipo “icómo?" "y" ¿porqué?", se tiene poco o (ningún) grado de control sobre el comportamiento real de los objetos/eventos en estudio y su } \\
\text { temporalidad es contemporánea" (Escudero et. al, 2008, p.10). } \\
\text { "... la investigación cualitativa es aplicable a una gran variedad de paradigmas de investigación. .. dentro de los cuales hay muchos métodos de investi- } \\
\text { gación, como lo son los estudios de casos, estudios de campo... En este trabajo analizamos la utilización de una técnica de investigación cualitativa muy } \\
\text { concreta: el estudio de casos..." (Cepeda, 2006, pp.58-59). } \\
\text { "This qualitative study investigated seven Singaporean in-service teachers' epistemic beliefs and their pedagogical beliefs. Two interviews were conducted } \\
\text { for each teacher. Employing a case study design, the transcripts were analyzed for assertions that reflected the teachers' beliefs. ... } \\
\text { The qualitative case study approach was adopted based on principles of constructivist inquiry (Guba y Lincoln, 1989) and a constant comparative method } \\
\text { wee employed for data analyses (Strauss y Corbin, 1990). The following sections provided the description of the context and research design" (Chai, 2010, } \\
\text { pp. 128, 130).". }\end{array}$ \\
\hline
\end{tabular}

Fuente: Elaboración propia. 
Al respecto Hernández et al (2006, p. 223-224) señala que para algunos autores y autoras los Estudios de Caso son considerados una clase de diseño de investigación (experimental, no experimental, cualitativo, etnográfico), o como un método, citando a Yin, 2003. "La realidad es que los estudios de caso son todo lo anterior. Poseen sus propios procedimientos y clases de diseños. Los podríamos definir como "estudios que al utilizar los procesos de investigación cuantitativa, cualitativa o mixta, analizan profundamente una unidad para responder al planteamiento del problema, probar hipótesis y desarrollar alguna teoría".

Sin embargo VanWynsberghe y Khan (2007, p. 3) cuestionan el referirse al EC como un método ${ }^{3}$ (un término que con frecuencia se confunde con metodología) pues podría implicar que el EC es una técnica, procedimiento o una forma para recolectar evidencias o datos. El EC no es un método porque los investigadores no pueden recopilar los datos obligatoriamente usando un único procedimiento; por el contrario en la investigación se emplean varios métodos de investigación que ayudan a construir o descubrir el caso. Además, a pesar de la existencia de diferentes tipos de caso de estudio (exploratorio, intrínseco, instrumental, etnográfico, longitudinal, por ejemplo), ninguno de ellos requiere un procedimiento específico de recolección de datos. Stake (2005, p. 443) al respecto aporta que el EC, como una forma de investigar, es definido por el interés en un caso individual, no por los métodos de indagación utilizados; el EC usa métodos pero el caso NO ES el método.

3. Para (Gurdián, 2010, p. 137) el método es una especie de brújula que evita que nos perdamos en el caos aparente de los fenómenos o las situaciones que investigamos; ayuda a no desviarse del camino a seguir e indica cómo plantear las preguntas de investigación o problemas, sin sucumbir ante las técnicas y los datos. También cumple con la función de solucionar problemas que emergen antes de, durante y posterior a la ejecución de una investigación
Al referirse a la posibilidad del EC como una metodología, VanWynsberghe y Khan (2007) señalan que este término -al igual que Estudio de Caso- es poco claro y se intercambia con frecuencia con el de método. Stake (2005, p. 443) indica que el EC no es una escogencia metodológica porque no define una forma de abordajemétodo de investigación, sino la escogencia de qué estudiar o documentar; de esta forma se asume que el investigador no escoge el caso; el proceso de investigación y, específicamente, la interacción entre caso y unidad de análisis, guía la elección de lo que será estudiado. Es por esto que el EC no es una metodología: no provee de una teoría detallada respecto a como debe desarrollarse la investigación, con métodos conceptualmente coherentes y acompañado de procedimientos de recolección de datos que se mapean desde la teoría.

El EC como estrategia implica la planificación de acciones en el tiempo para el logro de un determinado fin; desarrollando el proceso seleccionado para alcanzar un estado futuro. Dadas las características del EC en términos de su flexibilidad y su adecuación a partir del análisis de situaciones emergentes, no es posible referirse a este como una estrategia.

Sobre el EC como diseño de investigación $n^{4}$, VanWynsberghe y Khan (2007) retoman a Robert

4. Para Denzin y Lincoln (2005, p. 25) el diseño de la investigación_involucra un claro enfoque hacia la interrogante de la investigación, los propósitos del estudio, "qué información contestará las preguntas específicas de la investigación en forma más apropiada, y cuáles estrategias son más efectivas para obtenerla. Un diseño de investigación describe una serie flexible de pautas que conectan paradigmas teóricos primero a las estrategias de investigación, y segundo a los métodos para colectar materiales empíricos, situando al investigador en el mundo empírico y conectándolo con sitios específicos, personas, grupos, instituciones, y cuerpos de material interpretativo relevante, incluyendo documentos y archivos 
Yin (1994) (otra de las importantes figuras en el estudio de EC) para clarificar que estos se constituyen en un plan de acción que guía la investigación, desde las interrogantes básicas hasta las conclusiones e incluye los pasos para la recolección, análisis e interpretación de las evidencias a partir de proposiciones predefinidas, unidades de análisis, una lógica para enlazar los datos a las proposiciones y la aplicación de un set de criterios para la interpretación de los hallazgos. Así, si el EC fuera un diseño de investigación podría proveer a los investigadores de un plan prescriptivo, cosa que no cumple pues no ofrece tal guía de procedimientos para la recolección, análisis e interpretación de datos. Por esto no se considera como un diseño de investigación.

Ceballos (2009, p. 416) asume el "Estudio de Caso" como un enfoque de investigación desde el paradigma cualitativo-interpretativo que implica descripción, explicación y juicio respecto a la unidad de análisis. Concibe el proceso de investigación del EC de manera inductiva e identifica el conjunto de principios ontológicos, axiológicos, epistemológicos, metodológicos y retóricos que lo caracterizan y orientan.

\section{El concepto de Estudio de}

Caso en la Investigación Cualitativa

Podemos intentar ahora una propuesta de definición de Estudio de Caso en la Investigación Cualitativa (ECC).

El Estudio de Caso en la Investigación Cualitativa es un proceso de indagación focalizado en la descripción y examen detallado, comprehensivo, sistemático, en profundidad de un caso definido, sea un hecho, fenómeno, acontecimiento o situación particular. El análisis incorpora el contexto (temporo-espacial, económico, político, legal), lo que permite una mayor comprensión de su complejidad y, por lo tanto, el mayor aprendizaje del caso particular (ver figura 1).

\section{ESPIRAL DE PROFUNDIZACIÓN DE CONOCIMIENTO EN ESTUDIO DE CASO}

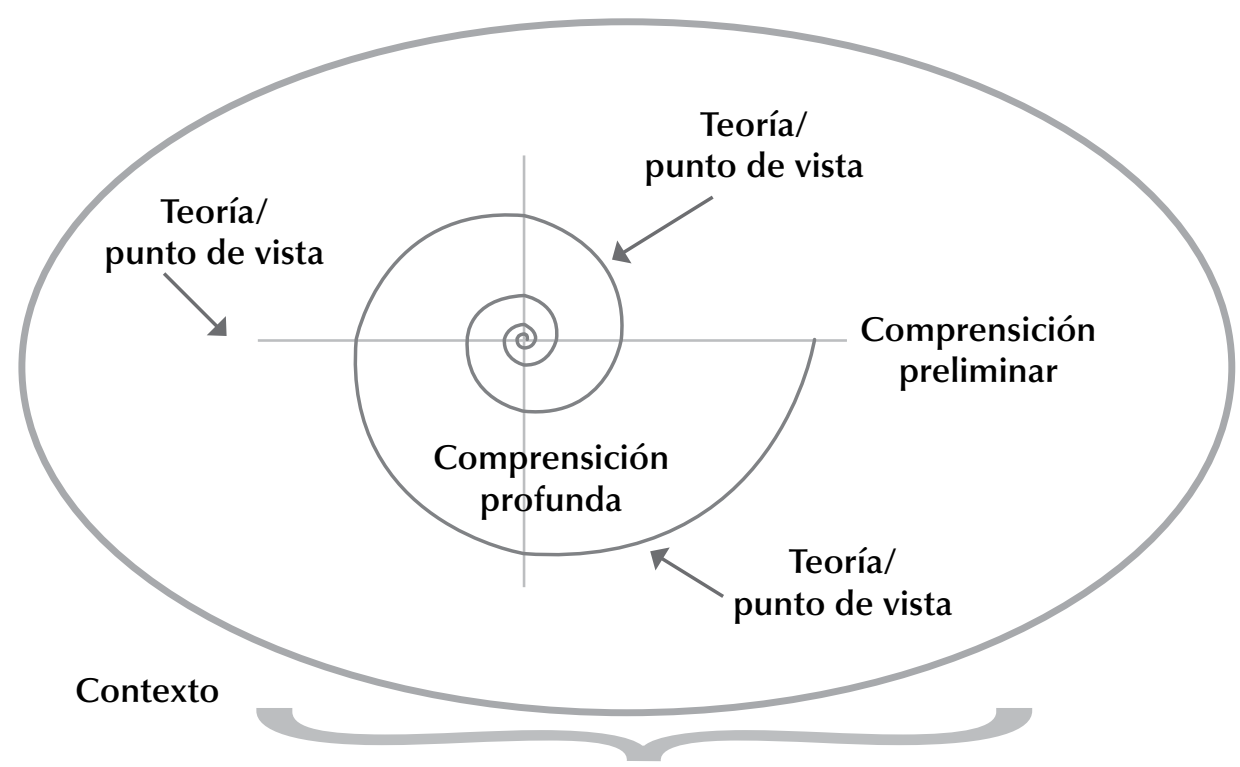

\section{Caso}

Elaboración propia, basada en Routio 2007. 
Dado que el ECC se define por el interés en un caso y no por los métodos de indagación utilizados ("el estudio de caso no es una elección metodológica, sino una elección de lo que será estudiado", Stake, 2005, p. 443) y que "no tiene especificidad, por lo que puede ser usado en cualquier disciplina para dar respuesta a preguntas de la investigación para la que se use"(Yin, 1993), es posible referirse a EC en cualquier disciplina: medicina, biología, derecho, antropología, física nuclear, informática, psicología y educación, por ejemplo.

Es además transparadigmático en el sentido de que puede utilizarse desde cualquier paradigma de investigación (teoría crítica, constructivismo, por ejemplo). Sin embargo el uso de múltiples métodos (triangulación) refleja un intento para obtener una profunda comprensión del fenómeno en cuestión convirtiéndose en una alternativa para la validación al añadir rigor, extensión, complejidad, riqueza, y profundidad a cualquier investigación en aras de alcanzar su objetivo: lograr el mayor conocimiento, integrado y holístico de un caso que genere oportunidades de aprendizaje (conocimiento experiencial).

El ECC puede usarse en cuatro diferentes situaciones (Yin, 2003 citado por Baxter y Jack, 2008): cuando el eje del estudio gira alrededor de respuestas a "cómo" y "por qué"; cuando la conducta involucrada en el estudio no puede ser manipulada; cuando se considera necesario analizar las condiciones contextuales porque son relevantes para el fenómeno en estudio; o cuando no hay una clara delimitación entre el fenómeno y el contexto.

Su unidad de análisis es el Caso, entendido como un fenómeno de algún tipo ocurrido en un contexto delimitado. Puede ser una persona, pareja, objeto, sistema, un acontecimiento particular o un hecho histórico, comunidad, departamento organización, un programa; la única exigencia es que posea algún límite físico, social o temporal que le confiera identidad. Tiene cuatro características principales: singularidad (particularidad), la complejidad, disponibilidad y potencial de aprendizaje.

1. La singularidad del caso refiere a aquello distintivo e importante que desde varios ángulos sólo entienden las personas próximas al caso y que lo diferencia de otros casos (Guba y Lincoln, 1985, Stake, 2005; Ceballos, 2009). González (2009) agrega que el caso debe tener elementos que llaman la atención por su novedad y extrañeza, de manera tal que el investigador recoja información pertinente para sistematizarla y profundizar en el conocimiento de los elementos intervinientes en el surgimiento de ese fenómeno o situación.

2. La complejidad se refiere al juego de interrelaciones políticas, económicas, sociales, culturales, personales, históricas, temporales y espaciales, que ocurren dentro del contexto (Yin, 2003; Stake, 2005; Ceballos, 2009).

3. Disponibilidad: los fenómenos se suceden de la dinámica cotidiana del desenvolvimiento humano, esto hace que algunos casos se presenten sin buscarlos y el investigador los aprovecha para iniciar su estudio sobre el mismo; por ejemplo cuando el miembro de un grupo adopta nuevos comportamientos o cuando se encuentra una pieza histórica única (González, 2009).

4. Potencial de aprendizaje: para Stake (2005) este aspecto es muy importante para la selección del caso y, en algunos casos, más relevante que la representatividad porque "es mejor aprender mucho de un caso atípico que un poco de uno típico" (p. 451). 
Stake (2005) señala cinco requerimientos para la optimización de la comprensión del caso:

1. Selección de tema/área, lo que es clave para el estudio. Deben considerarse los siguientes aspectos: determinar el caso identificando claramente la unidad de análisis; su delimitación, (puede ser por tiempo y lugar; tiempo y actividad; definición y contexto (Creswell, 2003; Stake, 1995; Miles y Huberman, 1994, citados por Baxter y Jack , 2008) conceptualizando el objeto de estudio, la selección del fenómeno, temas o áreas; tipo de caso: Yin categoriza los EC como explicativos, exploratorios o descriptivos; diferencia además entre individual, holístico y múltiple; según su propósito White (1992, citado por Stake, 2005) señala EC para identificación, explicación; control.

Según Stake (2005, pp.445-447) hay EC intrínsecos, instrumentales y colectivos o múltiples. el EC Intrínseco es aquel en el que se pretende alcanzar una mejor comprensión de un caso concreto, por un interés particular; este tiene importancia por sí mismo, por su particularidad, algo extraordinario, no es para entender un constructo abstracto, genérico o porque se pretenda construir teoría. El EC Instrumental es secundario en tanto que facilita la comprensión de algo, utilizándose para llegar a cierta conclusión o hacer generalizaciones. Puede ser típico o no y el investigador elige el caso en la medida en que aporte algo a la comprensión del tema objeto de estudio, de manera que este se convierte en herramienta -instrumento- para ilustrar un problema, una temática o un argumento. Un EC Colectivo o Múltiple se realiza cuando el interés se centra en un fenómeno, población o condición general; la indagación no es en un caso concreto sino en un determinado número de casos para su estudio intensivo. Se eligen varios casos de forma que ilustren situaciones extremas de un contexto bajo estudio; al maximizar sus diferencias, se evidencian las dimensiones del problema de forma clara. No se trata de acumular casos similares para aumentar la relevancia de posibles resultados; se trata de buscar casos lo más diferente posible en las dimensiones de análisis que, al menos en un primer momento, se consideran potencialmente relevantes.

2. La Triangulación $n^{5}$, refleja un intento para obtener una comprensión profunda del fenómeno en estudio. Stake (2005, p. 454) la define como "... un proceso de utilización de múltiples percepciones para aclarar significados, verificando la capacidad de repetición de una observación o interpretación", dado que la realidad objetiva nunca puede ser captada: conocemos una cosa sólo a través de sus representaciones. "La triangulación no es una herramienta o una estrategia de validación, sino una alternativa para la validación" (Flick, 2002, p. 227, citado por Denzin y Lincoln, 2005).

De esta forma también se procura reducir la probabilidad de malas interpretaciones, ambigüedades o confusiones y la defensa de múltiples perspectivas, ayudando a identificar diferentes realidades en torno al caso.

5 El término se tomó de la topografía y consiste en determinar ciertas intersecciones o coincidencias a partir de diferentes apreciaciones y fuentes informativas o varios puntos de vista del mismo fenómeno. En una investigación se pueden realizar varias "triangulaciones" para mejorar los resultados. Hay varios tipos básicos de triangulación: Triangulación de métodos y técnicas; Triangulación de datos; Triangulación de investigadores; Triangulación de teorías; Triangulación interdisciplinaria (Gurdián, 2010, p. 242) 
3. Conocimiento experiencial. Se refiere a la relación con la facilitación de experiencias vicarias - del investigador- y flexibilidad cognitiva -del lector-. Esto implica la forma de presentar la información como una indagación narrativa o contando una historia (storytelling). Lo constituye un conocimiento tácito, concreto e irreflexivo (que se va construyendo a partir de formas de actuación observadas, ...bien por procesos más o menos intuitivos de ensayo y error). Las concepciones implícitas más frecuentes suelen corresponderse con los estereotipos sociales dominantes aunque también poseen elementos creativos e intuitivos. El conocimiento experiencial lo componen además concepciones explícitas en las que está presente un cierto nivel de reflexión (conjunto de principios, concepciones, metáforas e imágenes que poseen las personas acerca de las diferentes variables presentes en su experiencia personal).

4. Contextos: deben considerarse aspectos históricos, culturales, físicos y geográficos, además de los contextos económico, social, político, ético y estético, en su interacción e influencia con el caso en estudio, según corresponda a la delimitación realizada.

5. Actividades: iniciar el estudio con un esbozo de idea de los que podría ser el reporte final, anticipando el orden y el tamaño de las partes de la historia. De esta forma se puede reducir las posibilidades de recuperar demasiados datos de cualquier tipo.

\section{CONCLUSIONES}

El Estudio de Caso es una forma de acercamiento a fenómenos complejos de la realidad, susceptibles de tratarse como sistemas abiertos que interactúan en su entorno, reconociendo el contexto y la interacción entre sus variables.

Posibilita la conexión entre investigación, teoría y práctica e induce a la acción en cuanto a que, dado que se inicia en la acción, las ideas que se van generando pueden ser utilizadas como retroalimentación, haciendo posible el descubrimiento de nuevas relaciones y conceptos. En este sentido es flexible. Además produce resultados que son asequibles a diferentes audiencias, incluso a públicos no especializados, por lo que su "consumo" puede ser inmediato.

Stake (2005) señala como clave para el trabajo en ECC que el investigador sea reflexivo (en el sentido de interpretativo), de manera que pueda trascender lo observacional hasta lograr integrar información y experiencias. Así, hay diferentes interpretaciones con diferentes perspectivas (fenomenológica, etnográfica ...).

Las críticas al ECC se ubican en cuatro aspectos fundamentales: 1. la confusión y pérdida de significados sobre su concepto, 2. la confiabilidad, 3. la validez y 4. la generalización de conocimiento. Para Flyvberg (2003) estos son cuestionamientos sobre el estatus mismo de los estudios de caso como método científico y que Flyvbjerg (Flyvbjerg, 2003; VanWynsberghe y Kahn, 2007) propone como mitos derivados.

Así, sobre el tema conceptual se han identificado abordajes del Estudio de Caso como enfoque, estrategia, método o metodología, diseño de investigación. Desde nuestra perspectiva, dadas las características del ECC, es un proceso de 
indagación multimetódico, transparadigmático y transdisciplinario que no debe encasillarse en ninguno de los conceptos anteriores.

Respecto a la confiabilidad y validez, la regulación del riesgo de imprecisión, falta de objetividad y rigor y consideraciones éticas (sesgos o problemas de prejuicios por ejemplo), coincido con Flyvberg (2003) en cuanto a que el ECC tiene su propio rigor, diferente, por supuesto, pero no menos estricto que el rigor de los métodos cuantitativos. La ventaja del ECC es que puede "acercarse" a situaciones de la vida real y comprobar ideas en relación directa con el fenómeno a medida que se desarrolla en la práctica. En este sentido la triangulación cobra gran importancia para minimizar estos problemas.

Sobre la generalización (referida a la aplicación de los hallazgos al resto de representantes que conforman el universo al que pertenece la muestra del estudio), Flyvberg (2003) señala que el ECC es ideal para la generalización utilizando el tipo de comprobación que Karl Popper ha llamado "falsación", que en la ciencia social forma parte de la reflexividad crítica, la reflexividad que Stake (2005) refiere en su sentido interpretativo, que permite trascender lo observacional hasta lograr integrar información y experiencias.

Parafraseando a Flyvberg (2003) y retomando a Stake (2005), un buen ECC se guía por el problema, no por la metodología, en tanto que emplea aquellos métodos que para una problemática dada, contribuyen mejor a contestar las preguntas de investigación planteadas. Allí esta su fuerza: en la profundización gracias a la triangulación, que permite el mayor aprendizaje posible del caso en estudio.

\section{REFERENCIAS}

Atwater, M.; Freeman, T.; Butler, M.; Draper-Morris, J. (2010). A Case Study of Science Teacher Candidates' Understandings and Actions Related to the Culturally Responsive Teaching of "Other". Students International Journal of Environmental and Science Education, v5 n3 p287-318 Jul 2010. Recuperado de http://www.eric. ed.gov/PDFS/EJ895740.pdf

Badilla, E. (2009). Diseño Curricular: de la Integración a la Complejidad. Revista Electrónica: Actualidades Investigativas en Educación, Volumen 9, Número 2, 2009, pp. 1-13. Instituto de Investigación en Educación, Universidad de Costa Rica. Recuperado de http:// revista.inie.ucr.ac.cr/articulos/2-2009/archivos/complejidad.pdf

Baxter, P.; Jack, S. (2008). Qualitative Case Study Methodology: Study Design and Implementation for Novice Researchers. Recuperado de http://www.nova.edu/ ssss/QR/QR13-4/baxter.pdf

Ceballos-Herrera, F. (2009). El informe de investigación con estudio de casos. Magis, Revista Internacional de Investigación en Educación. Vol 1 / Número 2 / pp 413-423, Enero-Junio 2009. Bogotá-Colombia / Página 413-423. Recuperado de http://www.javeriana.edu.co/magis/ numero-dos/PDFS-2/13-FCEBALLOS.pdf

Cepeda, C. (2006). La calidad en los métodos de investigación cualitativa: principios de aplicación práctica para estudios de casos. Cuadernos de Economía y Dirección de la Empresa. (ACEDE) n. 29. 057-082.

Chai, Ching Sing (2010). Teachers' Epistemic Beliefs and Their Pedagogical Beliefs: A Qualitative Case Study among Singaporean Teachers in the Context of ICT-Supported Reforms. Turkish Online Journal of Educational Technology - TOJET, v9 n4 p128-139 Oct 2010. Recuperado de http://www.eric.ed.gov/PDFS/ EJ908079.pdf

Denzin, N.; Lincoln, Y. (2005). The Sage Handbook of Qualitative Research. Third Edition. London, UK: Sage.

Ellet, W. (2007). The case study handbook. Harvard Business Scholl Press. Boston, Massachusetts. 
Escudero, J.; Delfín, L.; Gutiérrez, L. (2008). El estudio de caso como estrategia de investigación en las ciencias sociales. Ciencia Administrativa 2008-1. Recuperado de http://www.uv.mx/iiesca/revista/documents/estudio2008-1.pdf

Flyvbjerg, B. (2003). Cinco malentendidos acerca de la investigación mediante los estudios de caso. Recuperado de http://www.reis.cis.es/REIS/PDF/ REIS_106_041167998142322.pdf

González, L. (2009). Estudio de casos bajo el enfoque transdisciplinar. Multiciencias, Vol. 9, Núm. 3, septiembre-diciembre, 2009, pp. 303-312. Venezuela. Recuperado de http://redalyc.uaemex.mx/principal/ ForCitArt.jsp?iCve=90412325010\#

Guba, Y.; Lincoln, E. (1985). Naturalistic inquiry. Beverly Hills, CA: Sage.

Gurdián-Fernández, A. (2010). El paradigma cualitativo en la investigación socio-educativa. Editorial UCR

Hernández, R.; Fernández, C.; Baptista, P. (2006). Metodología de la Investigación. 4a edición. Editorial Mc Graw Hill: México.

Itard, J. M. G. (1801). De l'education d'un homme sauvage ou des premiers developpemens physiques et moraux du jeune sauvage de l'Aveyron. Goujon. Paris

Itard, J. M. G.: Rapports et memoires sur le sauvage de l'Aveyron. Traducción inglesa con introducción y notas de G. y M. Humprey: The wild boy of Aveyron. Century. New York, 1932. Traducción al castellano con introducción y notas de Rafael Sánchez Ferlosio: Víctor de l'Aveyron, Alianza, Madrid, 1982.

Marrero, M.; Woodruff, K.; Schuster, G.; Riccio, J (2010). Live, Online Short-Courses: A Case Study of Innovative Teacher Professional Development. International Review of Research in Open and Distance Learning, v11 n1 p81-95 Mar 2010. Recuperado de http://www. eric.ed.gov/PDFS/EJ881579.pdf
Mendoza, P; Berger, J. (2008). Academic Capitalism and Academic Culture: A Case Study. Education Policy Analysis Archives, v16 n23 Dec 2008. Recuperado de http://www.eric.ed.gov/PDFS/EJ826478.pdf

Onwuegbuzie, A; Leech, N. (2009). Generalization practices in qualitative research: a mixed methods case study. Quality y Quantity. Volume 44, Number 5, 881 892. Recuperado de http://www.springerlink.com/ content/0147037861125401/

Reis, M.; Cabral, L.; Peres, E.; Bessa, M.; Valente, A.; Morais, R.; Soares, S.; Baptista, J.; Aires, A.; Escola, J. J.; BulasCruz, J. A.; Reis, M. J. C. S. (2010). Using Information Technology Based Exercises in Primary Mathematics Teaching of Children with Cerebral Palsy and Mental Retardation: A Case Study. Turkish Online Journal of Educational Technology - TOJET, v9 n3 p106-118 Jul 2010. Recuperado de http://www.eric.ed.gov/PDFS/ EJ898019.pdf

Routio, P. (2007). Arteología. Estudio de casos. Helsinki. Recuperado de http://www2.uiah.fi/projects/metodi/

Shaw, C. (1931). The Natural History of a Delinquent Career. Chicago: The University of Chicago Press.

Siemens, L. (2010). The Potential of Grant Applications As Team Building Exercises: A Case Study. Journal of Research Administration, v41 n1 p75-91 Spr 2010. Recuperado de http://www.eric.ed.gov/ PDFS/EJ886794.pdf

Stake, R. (2007). Investigación con estudio de casos. $4^{a}$ reimpresión. Sage Publications, Inc. Recuperado de http://books.google.co.cr/books?id=gndJ0eSkGck Cypg=PA9ylpg=PA9ydq=robert+stake+estudio $+d$ $\mathrm{e}+$ casoysource $=$ blyots $=\mathrm{mORQ} 22 \mathrm{lH}$ olysig $=\mathrm{qCinOHo}$ TE_pcHzhFcDZ63ASMvVsyhl=esyei=hIJITe6FAsHLgQ e5rfWLBgysa=Xyoi=book_resultyct=resultyresnum = 9yved=0CFgQ6AEwCA\#v=onepageyqyf=false

Stake, R. (2005). Qualitative case studies. En N. K. Denzin y Y. S. Lincoln (Eds.), The SAGE handbook of qualitative research (pp. 443-466). Thousand Oaks, CA: Sage. 
RNA Revista Nacional de Administración

Stake, R. (1995). The Art Of Case Study Research.Thousand Oaks, Sage Publications Ed. Recuperado de http:// books.google.es/books?id=ApGdBx76b9kCyprintsec $=$ frontcover\#v=onepageyqyf=false

Stake, R. (1978). The Case Study method in social inquiry. Educational Researcher, Vol. 7, No. 3, Feb. 1978. Recuperado de http://www.ed.uiuc.edu/circe/Publications/1978_Stake.pdf

VanWynsberghe, R.; Khan, S. (2007). Redefining Case Study. International Journal of Qualitative Methods 6 (2) June 2007. Recuperado de http://www.ualberta. ca/ iiqm/backissues/6_2/vanwynsberghe.pdf

Yin, R. (2003). Case Study Research: Design and Methods. (3rd ed.). Thousand Oaks, CA: Sage.

Recibido: 20-01-2012

Aceptado: 08-03-2012

1343 (1), Enero - Junio, 2012 